\title{
UWB System Based on the M-OAM Modulation in IEEE.802.15.3a Channel
}

\author{
Khadija Hamidoun ${ }^{1}$, Raja Elassali ${ }^{1}$, Yassin Elhillali ${ }^{3}$, Khalid Elbaamrani ${ }^{1}$, A. Rivenq ${ }^{3}$, \\ and F. Elbahhar ${ }^{2}$ \\ ${ }^{1}$ ENSA, TIM, Marrakech, Morocco \\ ${ }^{2}$ IFSTAR, LEOST, F-59666 Villeneuve d'Ascq, \\ ${ }^{3}$ UVHC, IEMN-DOAE, F-59313 Valenciennes, France \\ k. hamidounegmail.com
}

\begin{abstract}
Known for many years but unexploited in the field of communications, ultra wideband systems (UWB) can be a solution to the saturation of the frequency bands. The advantage of such systems is that they are inexpensive in energy because of the limitations imposed by the standard. Our work is to design a high data rate IR-UWB system, through the use of modulation M-OAM (Orthogonal Amplitude Modulation), and the study of the system performances by adding the effect of the propagation channels dedicated to the UWB. The channel modeling is an important step in the implementation of any system, especially for those in development, as is the case of UWB systems. Although this model is one of the key elements of the overall chain system, it must take into account the distance between the two antennas, multipath and signal type. In this paper, we discover the existing models suitable for UWB channels and their implementation on our M-OAM system. The introduction of real channels in our chain of transmission reduces the quality of our system. Therefore, a RAKE receiver is proposed as a solution to improve performance.
\end{abstract}

Keywords: IR-UWB, channel effect, M-OAM, MGF, RAKE receiver.

\section{$1 \quad$ Introduction}

Recently, the main applications developed allowing the best use of the UWB spectrum, are: communication with high bit rate, low power, and short range, the second main applications are the communication with a low power and low bit rate [2]. As mentioned above, our study focuses on high-bit-rate systems, especially multimedia communication systems and intelligent transport such as vehicle to vehicle and vehicles to infrastructure applications [3].

The main purpose of our approach is to improve the quality of service of UWB communication systems, by using a high data rate modulation M-OAM (Orthogonal Amplitude Modulation). The IEEE.802.15.3a channel using M-OAM modulation is implemented and the perspective BER for each model is calculated. The performance is degraded with IEEE channels. By consequence, a RAKE receiver is proposed to circumvent this problem and improve performances. 
This paper is organized as follows. In the second section, we describe the orthogonal polynomials used in our UWB system. In this section, we focus on the properties of the modified Gegenbauer functions MGF. The third section presents an original high data rate modulation schemes called M-OAM based on orthogonal waveforms MGF. The main purpose of this paper is to evaluate the performances of our system in AWGN and IEEE.802.15.3a channels. Finally, the last section is dedicated to the study and implementation of RAKE receiver as a technique to improve the results. At the end, a conclusion is drawn with prospects.

\section{Collective Awareness System}

Our UWB M-OAM system is applied in the context of new information technologies related to transportation, in order to improve the quality of life of people by the collective awareness systems. Our application is included more precisely in the context of ITS (Intelligent Transportation System) that add some intelligence in transportation systems. Communication between vehicles would improve road safety, avoid traffic jams and reduce energy consumption, which is directly related to the release of gas $(\mathrm{CO} 2)$.

\section{Orthogonal Polynomials and M-OAM Modulations}

In this section, we present the Gegenbauer pulses as an example of orthogonal pulses that can be used for UWB communications. By definition, a family of polynomials $f_{n}(x)$ (where $n$ is the degree of the polynomial) is called orthogonal on the interval of definition $[a, b]$, if it satisfies the condition:

$$
\int_{a}^{b} w(x) f_{n}(x) f_{m}(x) d x=0
$$

Where $\mathrm{m}$ and $\mathrm{n}$ are non-negative integers unequal and $\mathrm{w}(\mathrm{x})$ is the weight of generalized scalar product [4]. Assuming that $\mathrm{w}(\mathrm{x})$ is strictly positive on the interval of definition. Equation (1) can be rewritten as a scalar product:

$$
\int_{a}^{b} f_{n}(x) f_{m}(x) d x=0
$$

Equation (2) ensures also that energy is normalized to unity, eg $1 \mathrm{~J}$, for each order of polynomial. Functions $\mathrm{f}_{\mathrm{n}}$ is then an orthonormal basis for a space of functions on the interval of definition $[a, b]$. A large number of families of polynomials can be considered, for example, the classical Hermite, Laguerre polynomials, Bessel and Gegenbauer polynomials. 


\subsection{Modified Gegenbauer Functions}

The Modified Gegenbauer functions (MGF) are waveforms based on orthogonal polynomials. These waveforms have been developed in IEMN_DOAE and IFSTARLEOST laboratories for communication applications [4] [5].

Gegenbauer polynomials are defined on the interval [-1,1] and satisfies the differential equation of second order defined as follows:

$$
G_{n, \beta}(x)=2\left(1+\frac{n+\beta-1}{n}\right) x G_{n-1, \beta}(x)-\left(1+\frac{n+2 \beta-2}{n}\right) G_{n-2, \beta}(x)
$$

The first 4 orders of these functions are defined in following equations and presented in figure 1:

$$
\begin{aligned}
G_{u}(0,1, x) & =1 *\left(1-x^{2}\right)^{1 / 4} \\
G_{u}(1,1, x) & =2 x *\left(1-x^{2}\right)^{1 / 4} \\
G_{u}(2,1, x) & =\left(-1+4 x^{2}\right) *\left(1-x^{2}\right)^{1 / 4} \\
G_{u}(3,1, x) & =\left(-4 x+8 x^{3}\right) *\left(1-x^{2}\right)^{1 / 4}
\end{aligned}
$$

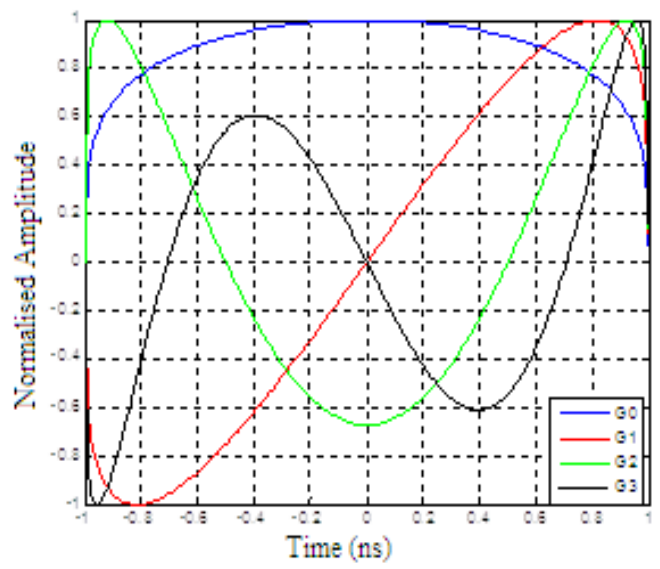

Fig. 1. The first 4 orders of Gegenbauer functions

These polynomials can be used in a UWB system to generate very short pulses. To do this, we propose to multiply $\mathrm{G}_{\mathrm{n}}(\boldsymbol{\beta}, \mathrm{x})$ by the square root of the weight function of this family of polynomials $\boldsymbol{w}(\boldsymbol{x}, \boldsymbol{\beta})$. [7] [8]

\subsection{M-OAM Modulation Schema}

The Orthogonal Amplitude Modulation (OAM) is the combination between the BiPhase Modulation (BPM) and Pulse Position Modulation (PPM) modulation using the orthogonal signal. In this section, we will see how the OAM is constructed by combaining the both modulation. 
The modulation 4-OAM transmits 2bits/symbol. This technique is the same as in the PPM bipolar; the first bit is represented by the position of the pulse and the second bit is represented by the phase (positive or negative) of the pulse. So, there are four possibilities combination of the pulse for this modulation. The symbol equation is:

With

$$
\mathrm{x}_{\mathrm{i}}(\mathrm{t})=\left(2 \mathrm{~S}_{\mathrm{iMSB}}-1\right) \mathrm{m}\left(\mathrm{t}+\left(2 \mathrm{~S}_{\mathrm{iLSB}}-1\right) \mathrm{T}\right)
$$

$$
\mathrm{m}(\mathrm{t})=2 \alpha \operatorname{Ktexp}\left(-\alpha \mathrm{t}^{2}\right)
$$

$S_{i}$ is the symbol sent, $m(t)$ the used waveform, T the time interval, LSB the Low Significant Bit and MSB the Most Significant Bit. Table 1 shows the 4-OAM pulse combination [7] [8].

Table 1. Symbols of 4-OAM modulation

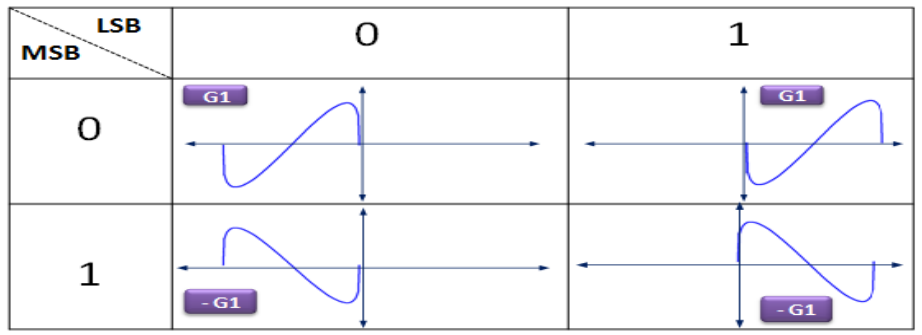

\section{$4 \quad$ Performances in IEEE.802.15.3a Channel}

At first, this section defines the characteristics of different IEEE 802.15.3a channels present in the standard. In a second step, we model a complete chain of our UWB system and evaluate its performances in terms of BER.

\subsection{Standard IEEE.802.15.3a}

IEEE 802.15.3a is based on the formalism of Saleh and Valenzuela; except that it is a real model and the amplitudes decay follows a law lognormal instead of an exponential decay, and also the first paths are not necessarily the strongest. [10] [11]

Parameters are defined to characterize the arrival of clusters $\Lambda$ and rays rates $\lambda$ as well as the coefficients of exponential decay inter-and intra-cluster $(\Gamma$ and $\gamma)$.

Four sets of model parameters are provided for four types of channels:

$>$ The channel model CM 1: corresponds to a distance of 0-4 $\mathrm{m}$ in the LOS;

$>$ The channel model CM 2: corresponds to a distance of $0-4 \mathrm{~m}$ in NLOS conditions;

$>$ The channel model CM 3: corresponds to a distance of 4 to $10 \mathrm{~m}$ in NLOS conditions;

> The channel model CM 4: corresponds to a NLOS situation with a large spread delay $\tau$ RMS $=25 \mathrm{~ns}$. 
This channel model has the merit of being a reference for studies of UWB systems. It applies in indoor environments at short range $(0$ to $10 \mathrm{~m})$. The following figures (Fig. 2 a-d) show the impulse responses of the four models.

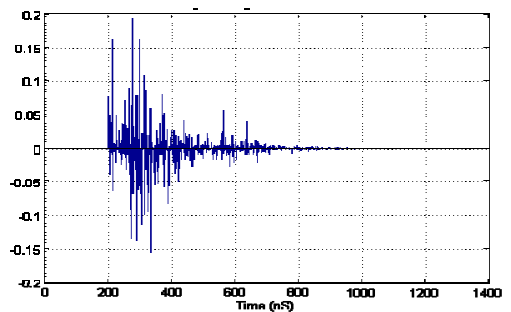

Fig. 2a. Impulse response of CM1

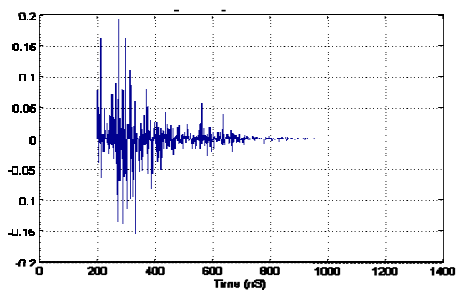

Fig. 2c. Impulse response of CM3

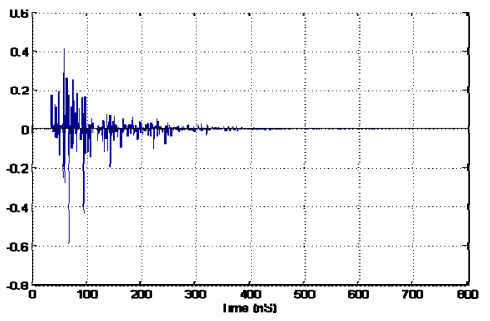

Fig. 2b. Impulse response of CM2

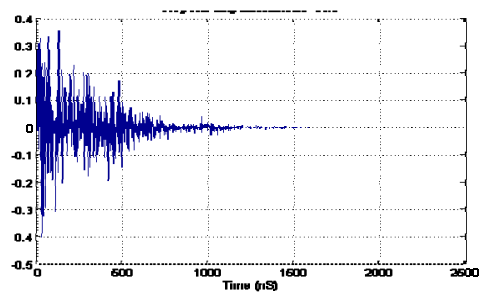

Fig. 2d. Impulse response of CM4

We note in the impulse response of CM1, that the delays have relatively low values, which makes sense since we're in a LOS (Line Of Sight) environment. For the last three models, we are in a NLOS (No Line Of Sight) environment, and we note here that the delays increase with the distance.

\subsection{Simulation Results}

To implement our UWB system, we simulated our transmission chain containing the transmitter using the 4-OAM modulations with MGF waveforms, the IEEE.802.15.3 channel according to the CM1-4 models, and finally, the demodulation at the reception. To examine the performance of our system, we calculated the BER versus SNR for each CM as well as for an AWGN channel, and then we compared the results. The Figures 3a-d show the BER versus SNR for each model.

Before the introduction of the channel, the BER was taking values less than $10^{-4}$ for a SNR greater than $10 \mathrm{~dB}$. While with the channel introduced, there is a degradation of the signal that can increase the value of BER up to $10^{-2}$ for the same SNR values chosen. By analyzing the graph of Figure 3, we see that the slope differs between AWGN and CM1-4, given the parameters used, namely the distance transmitter/receiver which is proportional to the BER, and the SNR that is inversely proportional to the AWGN and CM1-4. 
According to figure 2 and the simulation results, we deduce that CM1 offers the best conditions. However, with the other channels, we have more degraded performances. For that, we focus in future work on performance improvement solutions.

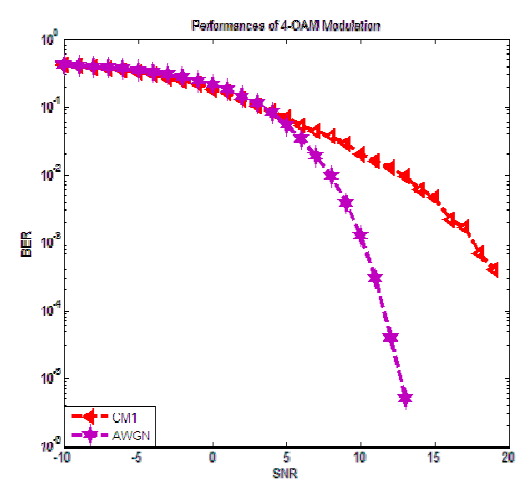

Fig. 3a. Performance in AWGN and CM1

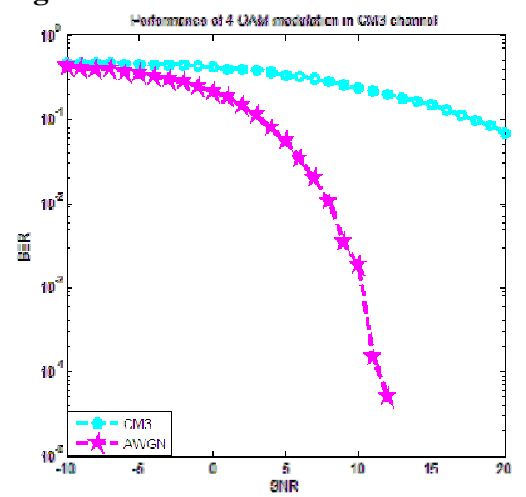

Fig. 3c. Performance in AWGN and CM3

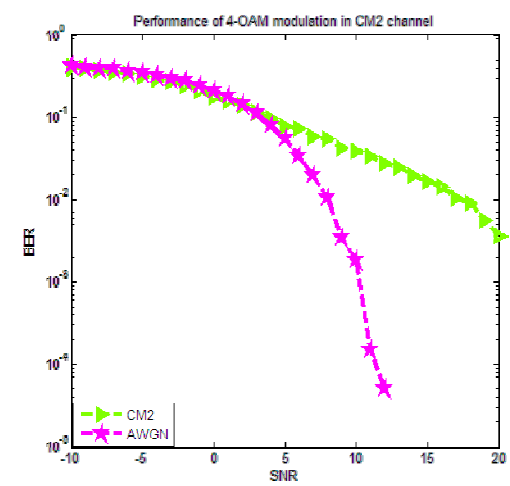

Fig. 3b. Performance in AWGN and CM2

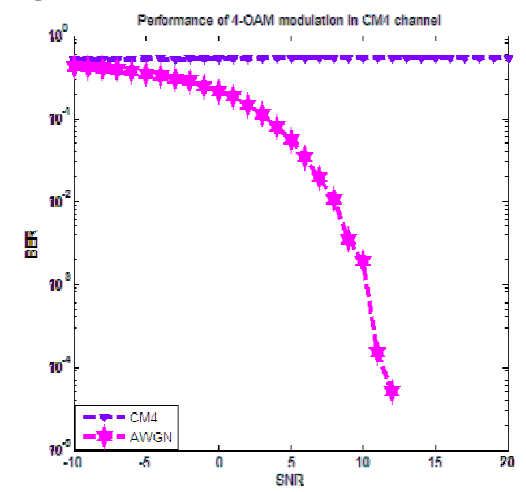

Fig. 3d. Performance in AWGN and CM4

\subsection{Performance Improvements}

The transmitted signal takes different paths due to the propagation channel. These multiple paths have different physical lengths, thus the signal from each trip comes with amplitude and delay specific to each path. These echoes induce a temporal spreading of the signal giving rise to a phenomenon of interference between transmitted symbols (ISI), which increases the bit error rate.

Several solutions have been proposed in the literature to combat this phenomenon. Among, there are the equalization techniques introduced at the reception, as well as the Rake receiver using multipath constructively. In this section, we tried to improve the results obtained above, by implementing the Rake receiver as one of the solutions.

To verify the results obtained after introduction of the Rake receiver, we chose to draw for each CM: one graph for AWGN, the second one for CM channel only, then introducing the RAKE receiver. Figures $4 \mathrm{a}-\mathrm{d}$ shows the simulation results. 


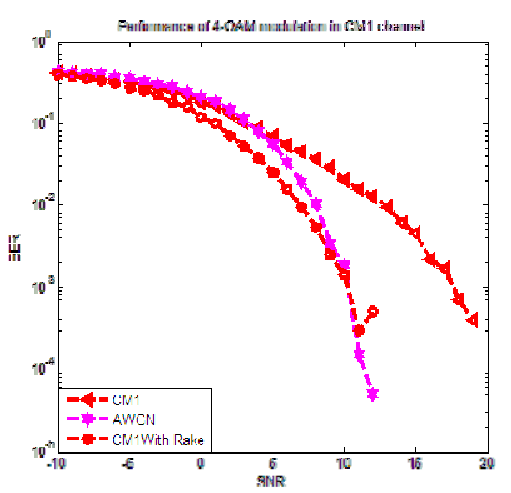

Fig. 4a. Comparison of CM1 and the Rake

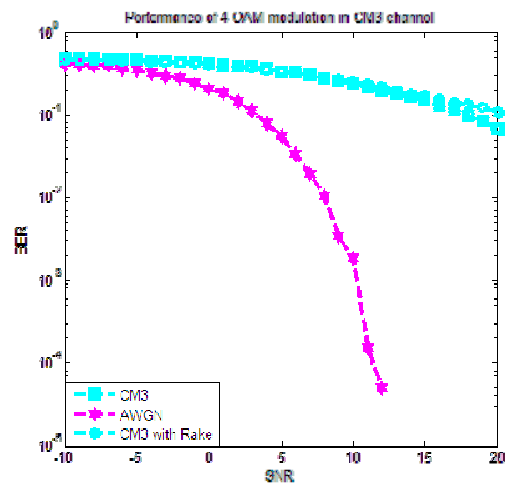

Fig. 4c. Comparison of CM3and the Rake

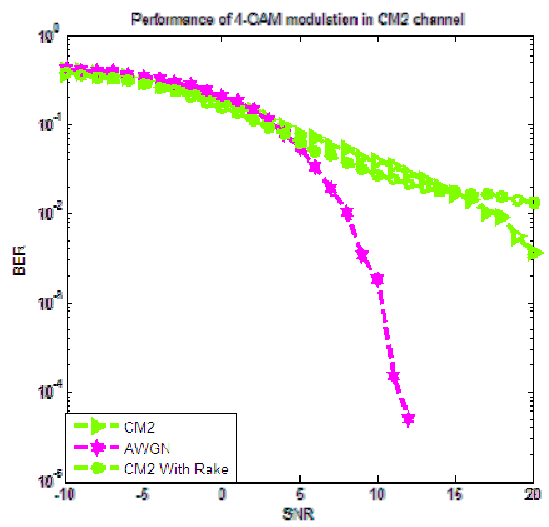

Fig. 4b. Comparison of CM2 and the Rake

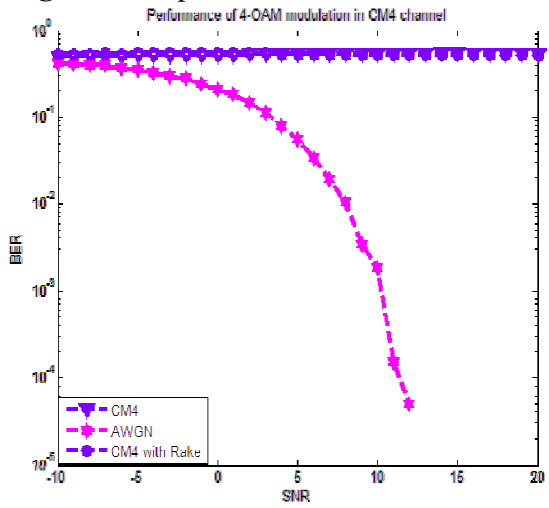

Fig. 4d. Comparison of CM4 and the Rake

The above figures show that:

- The implementation of the RAKE receiver after CM1 gives better results throughout the axis of SNR. Since we reached a BER of $0.03 \%$ for $11 \mathrm{~dB}$.

- The contribution of the RAKE receiver with CM1 gives more improvement than the CM2. For example for $\mathrm{SNR}=10 \mathrm{~dB}$, the BER has decreased from $3.9 \%$ to $2.7 \%$. From figure b, we see that for a SNR greater or equal to $15 \mathrm{~dB}$, there is no more improvement.

- The introduction of the Rake receiver with CM3 and CM4 channels (Figures 4.c and 4.d) has not led to a noticeable improvement in system performance compared to its absence. For CM3, 38\% of the BER were replaced by $36 \%$ for $\mathrm{SNR}=3 \mathrm{~dB}$. While for the CM4, we spent $54.5 \%$ to $51 \%$ for $\mathrm{SNR}=-1 \mathrm{~dB}$. This can be explained by the extreme conditions of the channel, which is a NLOS environment with a transmitter / receiver distance that exceeds $4 \mathrm{~m}$ for $\mathrm{CM} 3$ and $10 \mathrm{~m}$ for CM4. 


\section{Conclusion}

The objective of this work was the implementation of the 802.15.3a channel using 4OAM modulation and performances analysis of the system by calculating the BER. In conclusion, we noted that performances are degraded with IEEE channels, so we thought to include a solution to the reception, and we opted for the RAKE receiver.

The introduction of the Rake gave good results for the CM1 and average for the CM2. However, those of CM3 and CM4 were relatively weak for all values of SNR.

As perspective, the implementation of an equalizer will be very interesting and more specifically the MMSE. For best results, we aim to combine the MMSE with the RAKE receiver.

\section{References}

1. Shen, X., Guizani, M., Qiu, R.C., Le-Ngoc, T.: Ultra-wideband wireless communications and networks (2006)

2. Aiello, R., Batra, A.: Ultra wideband systems technologies and applications (2006)

3. Abed, E.: Etude et conception d'un système IR-UWB dédié aux communications sans fils haut débit, PhDthesis, Univ Valenciennes and Hainaut Cambrésis (January 2012)

4. Lamari, A.: Conception et modélisation d'un système de communication Multi-Utilisateurs basé sur la technique Ultra Large Bande, PhDthesis, University of Valenciennes and Hainaut Cambrésis (January 2007)

5. Elbahhar, F., Rivenq, A., Rouvaen, J.M.: Multi-user ultra-wide band communication system based on modified gegenbauer and hermite functions (2005)

6. Ghavami, M., Michael, L.B., Kohno, R.: UWB signals and systems in engineering

7. Abed, E.L., Elbahhar, F., Elhillali, Y., Rivenq, A., Elassali, R.: UWB communication system Based on Bipolar PPM with orthogonal waveforms, Wireless Engineering and Technology (2012)

8. Faten SALEM BAHRI: Contribution à l'étude des systèmes ultra wide band différentiels, $\mathrm{PhD}$ thesis, the national school of Telecommunications of Britain with the national school of engineers of Tunisia (May 2009)

9. Hamidoun, K., Elassali, R., Elhillali, Y., Elbahharr, F., Rivenq, A.: New adaptive architectures of coding and modulation UWB for infrastructure vehicle communication. In: 6th International Symposium on Signal, Image, Video and Communications, ISIVC 2012 (July 2012)

10. Cassioli, D., Win, M.Z., Molisch, A.F.: The Ultra-Wide Bandwidth Indoor Channel From Statistical Model to Simulations. IEEE JSAC 20, 1247-1257 (2002)

11. Cassioli, D., Win, M.Z., Molisch, A.F.: A statistical model for the UWB indoor channel. In: Proc. 53rd IEEE Vehicular Technology Conf., vol. 2, pp. 1159-1163 (May 2001) 